\title{
Through-pellicle inspection of EUV masks
}

\author{
Iacopo Mochi, Rajendran Rajeev, Patrick Helfenstein, Sara Fernandez, Dimitrios Kazazis \\ and Yasin Ekinci
}

Paul Scherrer Institut, 5232 Villigen, Switzerland

\begin{abstract}
RESCAN is a metrology platform, currently under development at Paul Scherrer Institut to provide actinic inspection capability for EUV reticles. It is a lensless microscope and its defect detection protocol is based on coherent diffraction imaging. One of the key features of an actinic pattern inspection tool is the ability to operate on reticles protected by an EUV pellicle. Thanks to the absence of imaging optics in close proximity of the sample, there are no geometrical constraints preventing the inspection of a pellicle-protected reticle in RESCAN. Nevertheless, the defect detection sensitivity depends on the quality of the reconstructed images and it is therefore important to assess if and how these are affected by the presence of an EUV pellicle. We report here the results of an evaluation of the effects of different types of EUV pellicles on the reconstructed images. We observed that high-absorption silicon nitride pellicles significantly reduce the imaging quality whereas in the case of the CNT-based pellicles the imaging performance was not affected. We also observed no damage of the CNT-based pellicle. To our knowledge, this work is the first successful attempt to perform mask inspection through EUV pellicles.
\end{abstract}

Keywords: EUV mask inspection, defect inspection, actinic, coherent diffraction imaging, EUV pellicle.

\section{INTRODUCTION}

Actinic pattern inspection (API) is an important cornerstone of the infrastructure required to support EUV lithography as it enters the high-volume manufacturing stage. Its role is to monitor the presence of defects on the reticle and assess their printability in different phases of the lithography process, including mask fabrication, exposure, and storage. An efficient API system should be able to detect and locate all the printable defects with high position accuracy in a short time. As for DUV reticles, EUV masks will eventually be equipped with protective pellicles to maximize the yield. [1,2] Although currently, a removable pellicle option is being considered, this is a risky and not ideal solution. Therefore, to be fully integrated into the lithography flow, an API system should also be capable of performing through-pellicle inspection.

The RESCAN tool (REflective-mode EUV mask SCANning microscope) is an actinic pattern inspection platform currently under development at Paul Scherrer Institute (PSI) [3-5]. It is installed at the XIL-II beamline of the Swiss Light Source. The beamline is equipped with a monochromator to guarantee a spectral bandwidth $\lambda / \Delta \lambda=1500$. The optical layout of RESCAN, outlined in Figure 1, consists of a variable aperture, a toroidal condenser, and a flat mirror that folds the beam towards the sample at an incidence angle of $6^{\circ}$. Both mirrors are coated with a Mo/Si multilayer to maximize the EUV reflectivity. Currently, RESCAN is hosted in a relatively small vacuum chamber that can accommodate samples as large as $2 \times 2 \mathrm{~cm}^{2}$ and the measurement area is limited to $200 \times 200 \mu \mathrm{m}^{2}$ which corresponds to the sample stage range.

RESCAN is a lensless scanning microscope based on coherent diffraction imaging (CDI). Compared with traditional EUV optical systems, CDI has the advantage of avoiding complex and expensive optics and their inherent physical limitations in terms of numerical aperture, resolution, and speed. Defect inspection is performed by reconstructing the sample's complex amplitude and comparing it with the reconstruction of a control region (die-to-die inspection) or with the complex amplitude calculated from the reticle design layout (die-to-database inspection). RESCAN has been successfully tested on logic pattern samples with programmed defects as small as $35 \times 141 \mathrm{~nm}^{2}$. Since the tool does not 
have any imaging optics, there is no physical obstacle to mount a pellicle on top of the sample. Nevertheless, it is necessary to verify the impact of the EUV membrane on the quality of the image reconstruction. For this purpose, we tested three different pellicles using a custom-made reticle sample and RESCAN's standard illumination conditions and we compared the reconstruction results to the data obtained on the same sample without the pellicle.

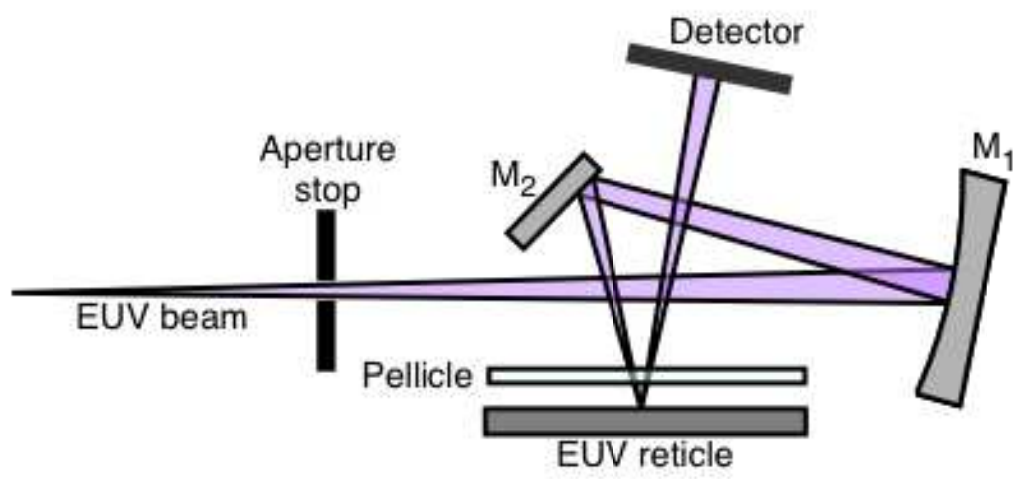

Figure 1. RESCAN optical layout. The EUV beam from the XIL-II beamline of the Swiss Light Source is focused by a toroidal condenser mirror $\left(\mathrm{M}_{1}\right)$ and folded onto the reticle at a $6^{\circ}$ angle by a flat mirror $\left(\mathrm{M}_{2}\right)$. The far-field diffraction signal is captured by a 2D EUV pixel detector.

\section{Sample reconstruction algorithm}

The CDI approach employed in RESCAN is an implementation of ptychography based on the difference map algorithm. [6] Ptychography is an iterative procedure capable of reconstructing the complex amplitude of the sample and of the illumination beam from a set of diffraction patterns intensity measurements. [7] This is achieved by scanning the sample with a step size smaller than the illumination probe. The redundancy thus generated in the data allows retrieving the phase information lost in the recorded diffraction patterns.

Two of the fundamental assumptions in standard ptychography implementations are that the illumination probe is constant and its location with respect to the sample is accurate. If these two conditions are violated, the algorithm may fail to converge. In general, the probe position with respect to the sample can vary with time because of mechanical vibrations or drifts and its intensity can be affected by misalignments and contamination of the illumination optics. It is possible to mitigate these effects with specific modifications to the reconstruction algorithm. [8] Ptychography iteratively searches for the complex amplitude of the object and of the illumination probe that are consistent with the recorded diffraction data. If the probe changes slowly during the scan of the sample, it is possible to let the algorithm pick different probes for different portions of the scan. $[9,10]$ This multi-probe approach mitigates the inconsistency induced in the data by long-period instabilities in the illumination intensity and position. If the probe changes on a time scale comparable with the single scan point measurement period, the reconstruction procedure can be modified with the statemixture algorithm. [11] In this algorithm, the illumination probe is modeled as the linear superposition of mutually orthogonal modes or states that describe the high-frequency vibrations and intensity changes of the illumination beam. As opposed to the multi-probe approach, which doesn't affect the speed of the reconstruction procedure, the statemixture algorithm requires a computation burden increase that is proportional to the number of modes used to model the probe.

\section{THROUGH-PELLICLE INSPECTION}

The through-pellicle inspection test consists in reconstructing a control sample and comparing the results to the ones obtained using a pellicle installed above the sample as shown in Figure 1. In the experiments described here, the pellicles were mounted statically at a distance $h=4 \mathrm{~mm}$ from the reticle. The reticle was free to move in its plane during the scan, 
but the pellicle remained fixed, so that the illumination beam hit always the same area on the membrane. The control sample consists of a silicon wafer substrate with a Mo/Si multilayer coating optimized for $6^{\circ}$ illumination and $\lambda=13.5 \mathrm{~nm}$.

The absorber stack is a single hydrogen silsesquioxane (HSQ) layer with a thickness of $140 \mathrm{~nm}$. The pattern was generated with e-beam lithography and consists of random structures with a critical dimension (CD) of $200 \mathrm{~nm}$ as shown in Figure 2.

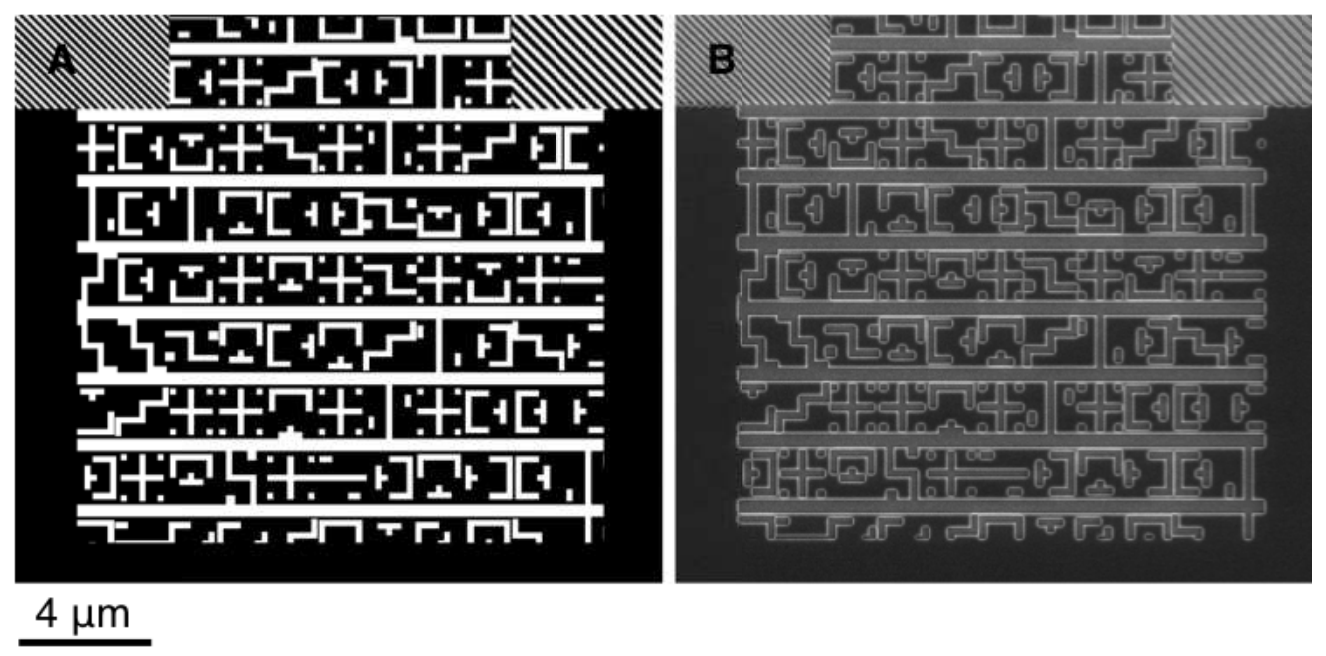

Figure 2. (A) GDS design layout and (B) SEM image of the EUV reticle.

RESCAN's illumination numerical aperture (NA) can be adjusted in discrete steps using a series of aperture stops with different radii. For these experiments we set it to its lowest value (0.002) to maximize the stability of the illumination intensity and to constrain the probe size on the sample. In RESCAN, the sample plane lies at a customizable distance from the illumination focus to control the beam footprint on the reticle. As the NA gets smaller, the probe intensity is less sensitive to drifts and vibrations of the beamline optics. This choice limits the data collection speed, requiring finer scan steps, but it proved to increase the reproducibility of the reconstruction, which is a critical factor in an image comparison analysis. The resulting beam footprint on the pellicle has a diameter $d=h \tan \left(\sin ^{-1}(N A)\right)=16 \mu \mathrm{m}$. The EUV flux density on the pellicle was estimated to be $\sim 3 \mathrm{~W} / \mathrm{cm}^{2}$.

The diffraction patterns generated by the reticle samples studied in RESCAN have a large dynamic range. This depends on the reflective nature of the samples, on the spatial frequency spectrum of the patterns, on the illumination settings used (small illumination NA), and on the necessity to collect high diffraction orders to achieve the highest possible resolution (large detector NA). The detector currently employed is an EUV CCD with a 16-bit ADC. To collect a fully resolved diffraction pattern, several images with different exposure times ranging from $0.1 \mathrm{~s}$ to $60 \mathrm{~s}$ are carefully combined together in a high dynamic range image, leading to total exposure times up to $130 \mathrm{~s}$ for each scan position.

An ideal EUV pellicle is a perfectly transparent film whose only effect is to isolate the reticle from the external environment. In practice though, any film will have a limited EUV transmission and will be subject to mechanical deformations or contaminations upon EUV exposure. This is particularly true for high-power scanners $(250 \mathrm{~W})$, but in our case, these effects can lead to time-dependent variations in the probe position and intensity that may affect the reconstruction quality. This effect manifested itself in the first through-pellicle experiment we performed using a 100$\mathrm{nm}$-thick $\mathrm{Si}_{3} \mathrm{~N}_{4}$ membrane. The membrane has an area of $2 \times 2 \mathrm{~mm}^{2}$, it is mounted on a silicon wafer frame and it has a nominal transmission of $42 \%$ in a single pass and $18 \%$ in a double pass [12].

We inspected the test sample through this pellicle with different total exposure times. In the absence of probe instabilities, we would expect to observe an increase in the reconstruction quality and resolution as we increase the exposure time and we improve the data signal-to-noise ratio, in agreement with the results obtained with a sample 
inspected without pellicle. A qualitative observation of the reconstructed object magnitude, shown in Figure 3, reveals, however, that the quality actually decreases with the exposure time. A total exposure time of $30 \mathrm{~s}$ shows already strong artifacts in the reconstructed object magnitude. Our hypothesis is that this effect is generated by the high absorption of the pellicle and the deformations induced by the local thermal load. These effects can be mitigated using the statemixture algorithm described in Section 1. We performed the reconstruction of the sample using the same dataset with 30 s exposure time, decomposing the probe on an orthonormal basis with three states (modes). The result, shown in Figure 2-D, demonstrates a significant reduction in the image artifacts and corroborates the hypothesis that the probe is being altered during the exposure by variations of the pellicle shape and properties.

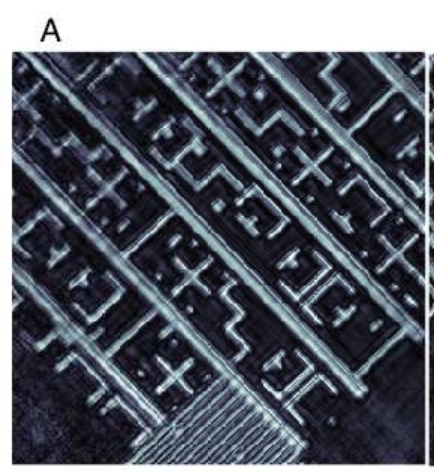

B

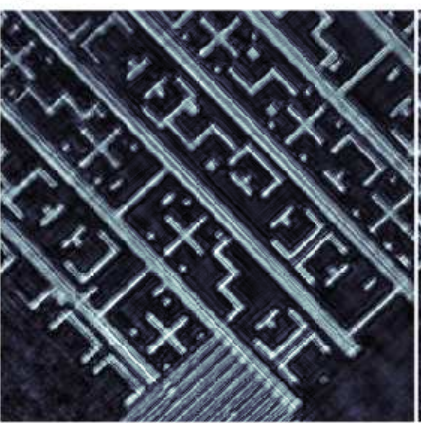

C

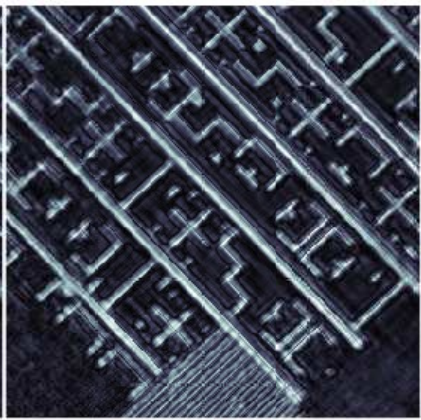

D

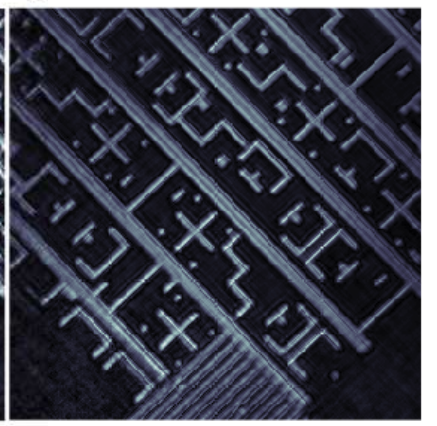

$2 \mu \mathrm{m}$

Figure 3. Reconstructed sample magnitude (normalized). A. Exposure time: 1 s. B. Exposure time: 5 s. C. Exposure time: 30 s. D. Exposure time: $30 \mathrm{~s}$ with state mixture correction.

\section{Through pellicle reconstruction performance}

We inspected the same area of the sample using three different pellicles: the 100-nm thick $\mathrm{Si}_{3} \mathrm{~N}_{4}$ pellicle described in the previous section, a single-walled carbon nanotube (SW-CNT) pellicle (EUV transmission 96\%) and a multi-walled aligned carbon nanotube (MW-CNT) one (EUV transmission 87\%). Both CNT-based pellicles had an area of $10 \times 10$ $\mathrm{mm}^{2}$ and were mounted on a silicon wafer frame. CNT pellicles have been proposed as an alternative to $\mathrm{Si}_{3} \mathrm{~N}_{4}$ and polysilicon membranes [13-14]. Their high transmission and low scattering [15] combined with their mechanical stress resilience [16], make them a promising candidate to be used for EUV lithography reticles. We used the three pellicles to collect a scan of our test reticle with the same experimental parameters and we compared the results to the magnitude of the reconstruction performed on the bare sample. In Figure 4 we show a qualitative comparison of the four cases where it is obvious that the two CNT pellicles have a very small impact on the image quality while the $\mathrm{Si}_{3} \mathrm{~N}_{4}$, as previously noted, induces strong artifacts in the sample image. It's important to note that the data collected through the CNT pellicles was not corrected with the state-mixture algorithm.

Each membrane was exposed to EUV light for a minimum of $6.8 \mathrm{~h}$ (total) inside the RESCAN chamber with an ambient pressure between $2 \cdot 10^{-8}$ and $2 \cdot 10^{-7}$ mbar and none of them showed any sign of post-exposure deterioration or contamination under visible light microscope inspection. 
A

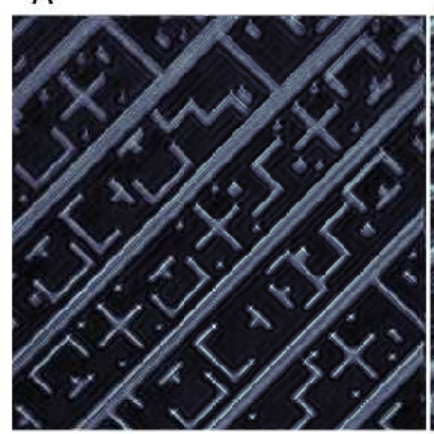

B

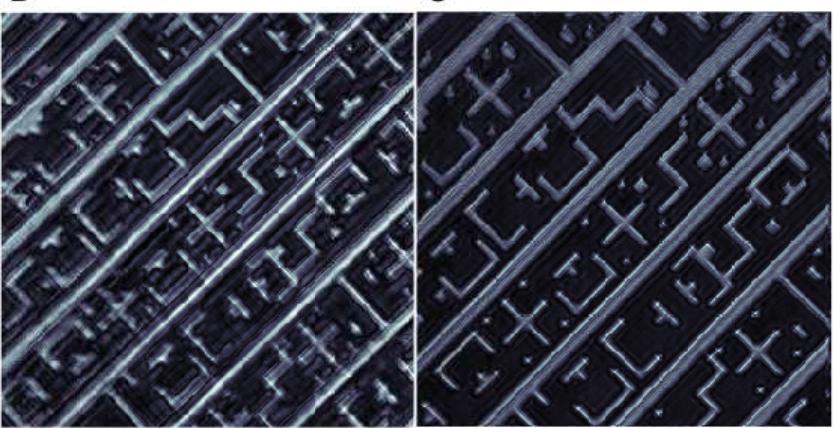

D

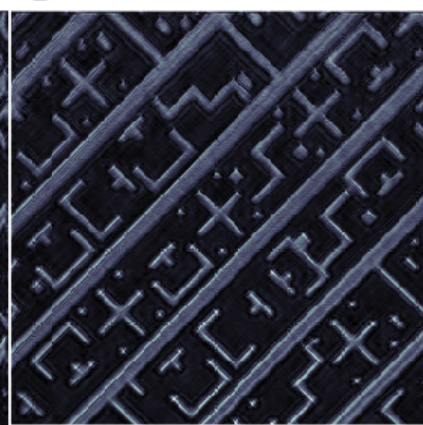

$\underline{2 \mu \mathrm{m}}$

Figure 4. Reconstructed sample magnitude (normalized) with (A) No pellicle, (B) with a $\mathrm{Si}_{3} \mathrm{~N}_{4}$ pellicle (thickness: $100 \mathrm{~nm}$ with $42 \%$ EUV transmission), (C) with a SW-CNT pellicle (96\% EUV transmission), and (D) with a MW-CNT pellicle ( $87 \%$ EUV transmission). None of these magnitudes were corrected with the state-mixture algorithm.

It is interesting to perform a quantitative evaluation of the impact of the pellicles on the quality of the sample's magnitude reconstruction. For this purpose, we define a simple image fidelity metric as the standard deviation of the difference between the reconstructed image magnitude obtained with the bare sample and the reconstructed image magnitude obtained with the different pellicles. In every experiment, the sample is reconstructed with an arbitrary position error. For this reason, before calculating the image error, a sub-pixel alignment was performed using a standard cross-correlation approach. The results are shown in Figure 5. In accordance with the qualitative observations above, the image fidelity error is lower for CNT pellicles in comparison to the $\mathrm{Si}_{3} \mathrm{~N}_{4}$ pellicle. Moreover, our add-on features implemented in the imaging algorithm (i.e. state-mixture correction) mitigate the deterioration of the images due to the $\mathrm{Si}_{3} \mathrm{~N}_{4}$ pellicle to a great extent but cannot correct it completely.

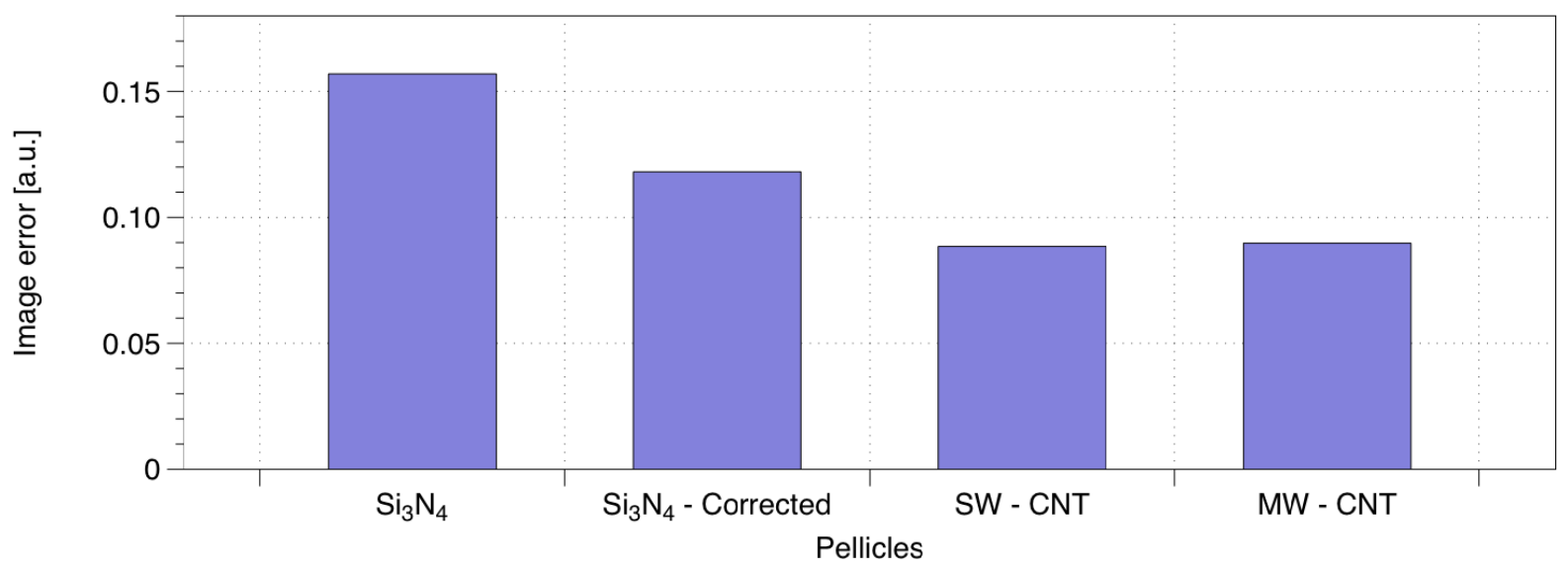

Figure 5. Image fidelity evaluation. The bars represent the standard deviation of the difference between the magnitude of the sample obtained without the pellicle and the ones collected with the different pellicles. Note that the state mixtures correction applied to the data collected with the $\mathrm{Si}_{3} \mathrm{~N}_{4}$ pellicle improves the image quality significantly, but the CNT pellicles perform better nonetheless.

\section{CONCLUSIONS AND OUTLOOK}

We tested the performance of RESCAN on samples protected with three different EUV membranes. RESCAN proved to be fully compatible with EUV pellicles from a mechanical perspective, thanks to the absence of optical or mechanical 
elements in close proximity to the surface of the sample. The three pellicles under test were mounted statically above the sample, which means that the illumination beam hit the pellicle surface always in the same location. We have not observed any visible exposure damage to the pellicles. In RESCAN, the defect detection is performed by comparing the complex amplitude of the sample with a defect-free version of it obtained from a control sample or derived computationally from the reticle layout; in either case, the quality of the reconstructed amplitude determines the defect sensitivity of the system. We estimated the impact of the different pellicles on the reconstruction fidelity by comparing the complex amplitude obtained from the bare sample to the ones obtained with the pellicles. Our analysis showed that the 100-nm-thick $\mathrm{Si}_{3} \mathrm{~N}_{4}$ pellicle induces severe artifacts in the reconstructed magnitude while both the SW-CNT and the MW-CNT pellicles don't alter the reconstruction visibly. We attribute this clear difference to the lower EUV absorption.

Furthermore, we proved that is possible to mitigate the artifacts in the reconstruction of the sample with the $\mathrm{Si}_{3} \mathrm{~N}_{4}$ membrane by decomposing the illumination in multiple orthogonal modes that model the alterations in the probe shape caused by the interaction between the illumination beam and the pellicle. Since RESCAN proved to be a versatile tool for EUV pellicle testing, we plan to continue this work including different pellicles, with and without coating, and to evaluate the effect of membrane-induced flare on defect sensitivity by using a specifically designed reticle sample with programmed defects and flare test patterns.

To our knowledge, this work is the first successful attempt to image through EUV pellicles, demonstrating both capabilities of RESCAN and the feasibility of CNTs as EUV pellicles. We note that the current experiments were performed without ambient hydrogen in the chamber. This will be a subject of future experiments.

\section{Acknowledgements}

We thank our colleagues at PSI Michaela Vockenhuber, Markus Kropf, José Gabadinho and Zuhal Tasdemir for their help and support. Special thanks to Emily Gallagher and Marina Timmermans from IMEC for providing the CNT pellicles, and to the membrane suppliers Canatu Oy and LINTEC.

\section{REFERENCES}

[1] Ron Hershel, "Pellicle Protection Of Integrated Circuit (IC) Masks," Proc. SPIE 0275, Semiconductor Microlithography VI, (28 July 1981).

[2] Ivan Pollentier, Jae Uk Lee, Marina Timmermans, Christoph Adelmann, Houman Zahedmanesh, Cedric Huyghebaert, Emily E. Gallagher, "Novel membrane solutions for the EUV pellicle: better or not?", Proc. SPIE 10143, Extreme Ultraviolet (EUV) Lithography VIII, 101430L (24 March 2017).

[3] Iacopo Mochi, Patrick Helfenstein, Rajendran Rajeev, Sara Fernandez, Dimitrios Kazazis, Shusuke Yoshitake, Yasin Ekinci, "Actinic inspection of EUV reticles with arbitrary pattern design", Proc. SPIE 10450, International Conference on Extreme Ultraviolet Lithography 2017, 1045007 (16 October 2017).

[4] Iacopo Mochi, Patrick Helfenstein, Istvan Mohacsi, Rajeev Rajendran, Dimitrios Kazazis, Shusuke Yoshitake, Yasin Ekinci, "RESCAN: an actinic lensless microscope for defect inspection of EUV reticles," Journal of Micro/Nanolithography, MEMS, and MOEMS 16(4), 041003 (27 July 2017).

[5] Patrick Helfenstein, Istvan Mohacsi, Rajendran Rajeev, Yasin Ekinci, "Scanning coherent diffractive imaging methods for actinic extreme ultraviolet mask metrology," Journal of Micro/Nanolithography, MEMS, and MOEMS 15(3), 034006 (16 September 2016).

[6] Pierre Thibault, Martin Dierolf, Oliver Bunk, Andreas Menzel, and Franz Pfeiffer. "Probe retrieval in ptychographic coherent diffractive imaging.". Ultramicroscopy, 2009 vol. 109 (4) pp. 338-343.

[7] Maiden, A. M., Rodenburg, J. M. "An improved ptychographical phase retrieval algorithm for diffractive imaging," Ultramicroscopy, 109(10), 1256-1262. (2009).

[8] A. M. Maiden, M. J. Humphry, M. C. Sarahan, B. Kraus, and J. M. Rodenburg, "An annealing algorithm to correct positioning errors in ptychography," Ultramicroscopy, vol. 120, pp. 64-72, Sep. 2012

[9] M. Odstrcil et al., "Ptychographic coherent diffractive imaging with orthogonal probe relaxation," Opt. Express, vol. 24, no. 8, pp. 8360-8369, Apr. 2016.

[10] P. Helfenstein, R. Rajeev, I. Mochi, A. Kleibert, C. A. Fernandez Vaz, and Y. Ekinci, (2018). "Coherent Diffractive Imaging of Photomasks for Extreme Ultraviolet Lithography", Manuscript submitted for publication. 
[11] Thibault, P., Menzel, A. (2013). Reconstructing state mixtures from diffraction measurements. Nature, 494(7435), 68-71.

[12]B.L. Henke, E.M. Gullikson, and J.C. Davis. X-ray interactions: photoabsorption, scattering, transmission, and reflection at E= 50-30000 eV, Z=1-92, Atomic Data and Nuclear Data Tables Vol. 54 (no.2), 181-342 (July 1993).

[13] Marina Y. Timmermans, Ivan Pollentier, Jae Uk Lee, Johan Meersschaut, Olivier Richard, Christoph Adelmann, Cedric Huyghebaert, Emily E. Gallagher, "CNT EUV pellicle: moving towards a full-size solution", Proc. SPIE 10451, Photomask Technology, 104510P (16 October 2017)

[14] Ivan Pollentier, Johannes Vanpaemel, Jae Uk Lee, Christoph Adelmann, Houman Zahedmanesh, Cedric Huyghebaert, Emily E. Gallagher, "EUV lithography imaging using novel pellicle membranes", Proc. SPIE 9776, Extreme Ultraviolet (EUV) Lithography VII, 977620 (18 March 2016)

[15]Frank Scholze, Christian Laubis, Michael Krumrey, Marina Y. Timmermans, Ivan Pollentier, Emily E. Gallagher, "EUV optical characterization of alternative membrane materials for EUV pellicles ", Proc. SPIE 10451, Photomask Technology, 104510R (16 October 2017)

[16] Jae Uk Lee, Johannes Vanpaemel, Ivan Pollentier, Christoph Adelmann, Houman Zahedmanesh, Cedric Huyghebaert, Marina Timmermans, Michael De Volder, Emily Gallagher, "Introducing the EUV CNT pellicle", Proc. SPIE 9985, Photomask Technology 2016, 99850C (25 October 2016) 